Invited review

\title{
Fasciola hepatica cathepsin L-like proteases: biology, function, and potential in the development of first generation liver fluke vaccines
}

\author{
John P. Dalton ${ }^{\mathrm{a}, \mathrm{b}, *}$, Sandra O. Neill ${ }^{\mathrm{a}, \mathrm{c}}$, Colin Stack ${ }^{\mathrm{a}, \mathrm{d}}$, Peter Collins $^{\mathrm{a}}$, Alan Walshe ${ }^{\mathrm{a}}$, \\ Mary Sekiya $^{\mathrm{a}}$, Sean Doyle ${ }^{\mathrm{d}}$, Grace Mulcahy ${ }^{\mathrm{b}, \mathrm{e}}$, Deborah Hoyle ${ }^{\mathrm{f}}$, Eric Khaznadji ${ }^{\mathrm{g}}$, \\ Nathalie Moirég , Gerard Brennan ${ }^{\mathrm{h}}$, Angela Mousley ${ }^{\mathrm{h}}$, Natalia Kreshchenko ${ }^{\mathrm{h}}$, \\ Aaron G. Maule ${ }^{\mathrm{h}}$, Sheila M. Donnelly ${ }^{\mathrm{a}}$ \\ ${ }^{a}$ Molecular Parasitology Unit, School of Biotechnology, Dublin 9, Ireland \\ ${ }^{\mathrm{b}}$ Ildana Biotech, INVENT, Dublin City University, Glasnevin, Dublin 9, Ireland \\ ${ }^{\mathrm{c}}$ School of Nursing, Dublin City University, Dublin 9, Ireland \\ ${ }^{\mathrm{d}}$ Department of Biology, National University of Ireland, Maynooth, Co. Kildare, Ireland \\ ${ }^{\mathrm{e}}$ Department of Veterinary Microbiology and Parasitology, Faculty of Veterinary Medicine, University College Dublin, Belfield, Dublin 4, Ireland \\ ${ }^{\mathrm{f}}$ Centre for Tropical Veterinary Medicine, Royal (Dick) School of Veterinary Studies, University of Edinburgh, Roslin, Midlothian EH25 9RG, Scotland, UK \\ gINRA, UR86 “Bio-Agresseurs, Santé et Environnement", 37380 Nouzilly, France \\ ${ }^{\mathrm{h}}$ School of Biology and Biochemistry, The Queen's University of Belfast, Medical Biology Centre, 97 Lisburn Road, Belfast BT9 $7 B L$, UK
}

Received 11 November 2002; received in revised form 15 May 2003; accepted 16 June 2003

\begin{abstract}
Fasciola hepatica secretes cathepsin L proteases that facilitate the penetration of the parasite through the tissues of its host, and also participate in functions such as feeding and immune evasion. The major proteases, cathepsin L1 (FheCL1) and cathepsin L2 (FheCL2) are members of a lineage that gave rise to the human cathepsin Ls, Ks and Ss, but while they exhibit similarities in their substrate specificities to these enzymes they differ in having a wider $\mathrm{pH}$ range for activity and an enhanced stability at neutral $\mathrm{pH}$. There are presently 13 Fasciola cathepsin L cDNAs deposited in the public databases representing a gene family of at least seven distinct members, although the temporal and spatial expression of each of these members in the developmental stage of $F$. hepatica remains unclear. Immunolocalisation and in situ hybridisation studies, using antibody and DNA probes, respectively, show that the vast majority of cathepsin L gene expression is carried out in the epithelial cells lining the parasite gut. Within these cells the enzyme is packaged into secretory vesicles that release their contents into the gut lumen for the purpose of degrading ingested host tissue and blood. Liver flukes also express a novel multi-domain cystatin that may be involved in the regulation of cathepsin L activity. Vaccine trials in both sheep and cattle with purified native FheCL1 and FheCL2 have shown that these enzymes can induce protection, ranging from 33 to $79 \%$, to experimental challenge with metacercariae of $F$. hepatica, and very potent anti-embryonation/hatch rate effects that would block parasite transmission. In this article we review the vaccine trials carried out over the past 8 years, the role of antibody and $\mathrm{T}$ cell responses in mediating protection and discuss the prospects of the cathepsin Ls in the development of first generation recombinant liver fluke vaccines.
\end{abstract}

(C) 2003 Australian Society for Parasitology Inc. Published by Elsevier Ltd. All rights reserved.

Keywords: Helminths; Trematodes; Parasites; Cathepsins; Proteases; Vaccines; Immunology; Biochemistry

\section{Introduction}

The trematode parasites Fasciola hepatica (temperate) and Fasciola gigantica (tropical) are the causative agents of liver fluke disease (fasciolosis) in cattle and sheep. Infection

\footnotetext{
* Corresponding author. Tel.: + 353-1-7005407; fax: + 353-1-7005412.

E-mail address: john.dalton@dcu.ie (J.P. Dalton).
}

causes world-wide economic losses of approximately US\$2,000 million annually to the agricultural sector (Spithill et al., 1999a). Fasciolosis is also an emerging pathogen of humans particularly in the South American countries Bolivia, Peru and Equador, in Egypt and Iran (O'Neill et al., 1998; Mas-Coma et al., 1999; Rokni et al., 2002). It is estimated that 2.4 million people are infected with liver fluke world-wide (Mas-Coma et al., 1999). 
Fasciolosis is acquired following the ingestion of vegetation or water contaminated with the encysted infectious liver fluke larvae, known as metacercariae. Metacercariae excyst in the intestines, burrow through the intestinal wall and migrate into the liver tissue where they spend about 8-12 weeks feeding on host tissue and blood and consequently causing extensive haemorrhaging and perforations. This acute stage of disease can result in death in highly infected sheep, but death is rarely seen in cattle. After this period the parasites move into the bile ducts where they complete their growth and maturation. The hermaphroditic adult liver flukes puncture the wall of the bile duct and feed on blood that provides the nutrient for the production of enormous numbers of eggs that are carried into the intestine with the bile fluids and are passed onto pasture with the faeces. An aquatic larval stage hatches from the eggs and infects an intermediate mud-snail host, such as Lymnaea truncatula. After several developmental and multiplication stages within the snail the parasites emerge and become encysted on vegetation and thus continue the cycle (Andrews, 1999).

A number of chemicals are commercially available for the treatment of animal fasciolosis, including closantal, clorsulon, rafoxanide, nitroxynil and triclabendazole (see Fairweather and Boray, 1999). Following dosing, the therapeutic concentration of these drugs in the blood is reached for approximately 1-2 days and hence only current parasitic infections are cleared. Animals on contaminated pastures are then susceptible to re-infection. Triclabendazole requires particular mention since this is the only drug effective against early stage parasites, i.e. the stages that migrate through the liver and induce acute fasciolosis. The drug was launched in Ireland in 1983 under the brand name Fasinex (Ciba-Geigy, now Novartis Animal Health) and has enjoyed substantial commercial success. However, in 1995 an anonymous letter to the Irish Farmer's Journal (Anon., 1995) reported the presence of triclabendazole-resistant liver flukes on a farm in Co. Sligo, an area where fasciolosis is endemic. If verified this provides an example of how quickly (within 12 years) drug resistance to anthelminthics can appear in the field. Triclabendazole resistance has now been reported in Australia (Overend and Bowen, 1995; Fairweather and Boray, 1999), Scotland (Mitchell et al., 1998) and the Netherlands (Moll et al., 2000; Gaasenbeek et al., 2001).

Apart from the appearance of drug resistant parasites, other pressures in the form of EU regulations, consumer concerns for 'greener' food and for animal welfare, and environmental issues regarding the passage of chemical residues onto pastures and into waterways will make the control of helminth diseases, including fasciolosis, with chemical drugs unsustainable. The future of liver fluke control will almost certainly depend on the development of a protective vaccine; the challenge will be to develop and commercialise such a product that will give a benefit that is visible to farmers (i.e. enhanced performance of animals, reduced deaths, etc.) at a competitive price (i.e. at a cost as low as drug treatments). It must be remembered, however, that vaccines provide 'protection' against infection and thereby have an advantage over drugs (particularly those that are effective against the adult liver fluke stages alone) that only provide a 'treatment'. Moreover, vaccines are considered safe and environmentally friendly.

Recent advances in molecular biology (proteomics and genomics) have made it relatively easy to identify and isolate potential vaccine components but in the case of liver flukes there is still a dearth of vaccine candidates that have been tried and tested in the target species, i.e. sheep and cattle. Research on a number of molecules including cathepsin Ls, glutathione $S$-transferase (GST), leucine aminpeptidase (LAP) and fatty acid binding proteins (FABP), however, has demonstrated the feasibility of inducing protective responses in laboratory and large animal models (reviewed by Spithill and Dalton, 1998; Spithill et al., 1999a). This article will review the work of our laboratory and others on liver fluke cathepsin L proteases and update the progress towards a vaccine.

\section{Biochemical characteristics of cathepsin Ls}

Fasciola hepatica cathepsin L proteases were the first of this class of enzyme to be described in helminths (Smith et al., 1993). It is now known that this enzyme class is expressed by all parasitic worms and in many cases they are secreted and hence are pivotal in the host-parasite interplay. However, they appear to be particularly prominent in trematodes as compared to nematodes where cathepsin B-like enzymes tend to be expressed in greater amounts (Tort et al., 1999).

Enzyme activity attributable to cysteine proteases was detected in our laboratory in extracts of $F$. hepatica parasites and ES products over a decade ago (Dalton and Heffernan, 1989). However, a more recent characterisation of these extracts using synthetic peptide substrates, such as Z-Phe-Arg-NHMec, demonstrated that the dominant activity was cathepsin L-like. Two distinct cathepsin L proteases have been purified in our laboratory from culture medium in which adult $F$. hepatica parasites were incubated and shown to be the predominant proteolytic activity secreted by the worms (Smith et al., 1993; Dowd et al., 1994). Subsequently, transcripts encoding these two major cathepsin L-like proteases, termed FheCL1 and FheCL2, have been isolated from cDNA libraries by immunoscreening with anti-sera prepared against the native enzymes (Dowd et al., 1997; Roche et al., 1997). These transcripts encode zymogens with structures that are similar to those of vertebrate cathepsin Ls in that they consist of a hydrophobic signal peptide (12-20 residues), a pro-segment (100 residues) and a mature enzyme (200 residues) (see Fig. 1). Unlike mammalian cathepsin Ls, FheCL1 and FheCL2 do not possess potential $N$-glycosylation sites in their mature 


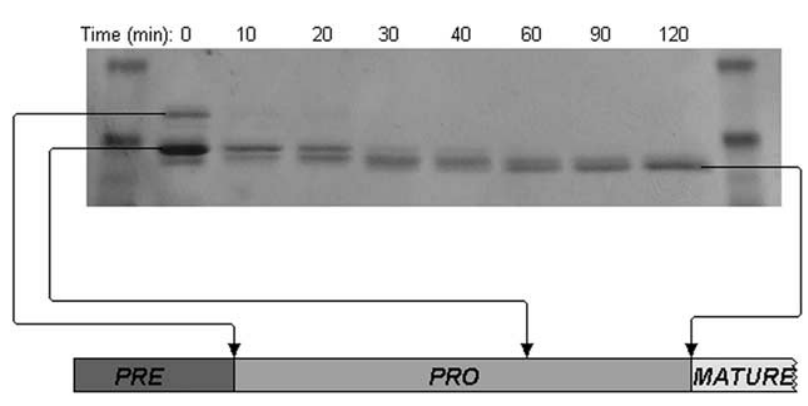

Fig. 1. Activation of Ni-NTA-agarose purified P. pastoris-expressed recombinant FheCL1 at pH 5.5. Purified recombinant FheCL1 resolves as two bands of 38 (upper) and $34 \mathrm{kDa}$ (lower, arrowed) that represent the full pre-pro-mature and a truncated pro-mature form of the enzyme, respectively. While this preparation exhibits cathespin L activity it can be further activated to the fully processed mature enzyme (last lane on right) by incubating it for a period of $1-2 \mathrm{~h}$ in $0.1 \mathrm{M}$ sodium acetate, $\mathrm{pH}$ 5.5. The positions of the major proteins resolved in the gel with respect to the cathepsin L pro-enzyme are shown schematically and were determined by $\mathrm{N}$-terminal sequencing.

enzyme portion and electrophoretic/immunoblot analyses suggest that they are not glycosylated.

The substrate specificity of the cathepsin Ls was examined using synthetic fluorogenic peptide substrates that are classically used to characterise mammalian cathepsin Ls, Ss, Ks (Z-Phe-Arg-NHMec), cathepsin B (Z-Arg-Arg-NHMec) and cathepsin H (Z-Arg-NHMec) (Barrett and Kirschke, 1981). Both enzymes demonstrated high catalytic efficiency $\left(k_{\text {cat }} / K_{\mathrm{m}}\right)$ for Z-Phe-Arg-NHMec, but showed little or no activity against the latter two substrates. This substrate specificity is consistent with their grouping with the non-cathepsin B proteases; however, FheCL2 could also cleave substrates that contained proline in the $\mathrm{P}_{2}$ position, and the substrate Z-Gly-Pro-Arg is used to distinguish between FheCL1 and FheCL2 activities (Dowd et al., 1994, 1997).

The $F$. hepatica cathepsin L-like proteases are active over a wide $\mathrm{pH}$ range $(\mathrm{pH} 3.0-8.0)$, and stability studies demonstrated that FheCL1 retains $100 \%$ of its activity when incubated at $37{ }^{\circ} \mathrm{C}$ for $24 \mathrm{~h}$. In contrast, human cathepsin $\mathrm{L}$ is irreversibly inactivated above $\mathrm{pH}$ 7.0, a property that is thought to protect cells against uncontrolled proteolysis in the event of accidental leakage from the lysosomes (Dowd et al., 2000). The stability of the liver fluke enzymes may reflect the fact that the parasite employs them to generate a migratory path through a large tissue mass, the liver, on its way to the bile ducts.

\section{The cathepsin $L$ gene family}

Early studies by Heussler and Dobbelaere (1994) indicated that $F$. hepatica expressed several cathepsin $\mathrm{L}$ genes, but since this report the number of cathespin L cDNA entries into the public databases has gradually grown so that at writing there are 13 known $F$. hepatica cathepsin genes. The number of $F$. gigantica cathepsin $\mathrm{L}$ entries in the databases has also grown mainly due to the efforts of Grams et al. (2001) and Yamasaki et al. (2002), and unsurprisingly many of these genes appear to be homologues of the $F$. hepatica genes. The family as it stands is presented in Fig. 2; some of the encoded polypeptides

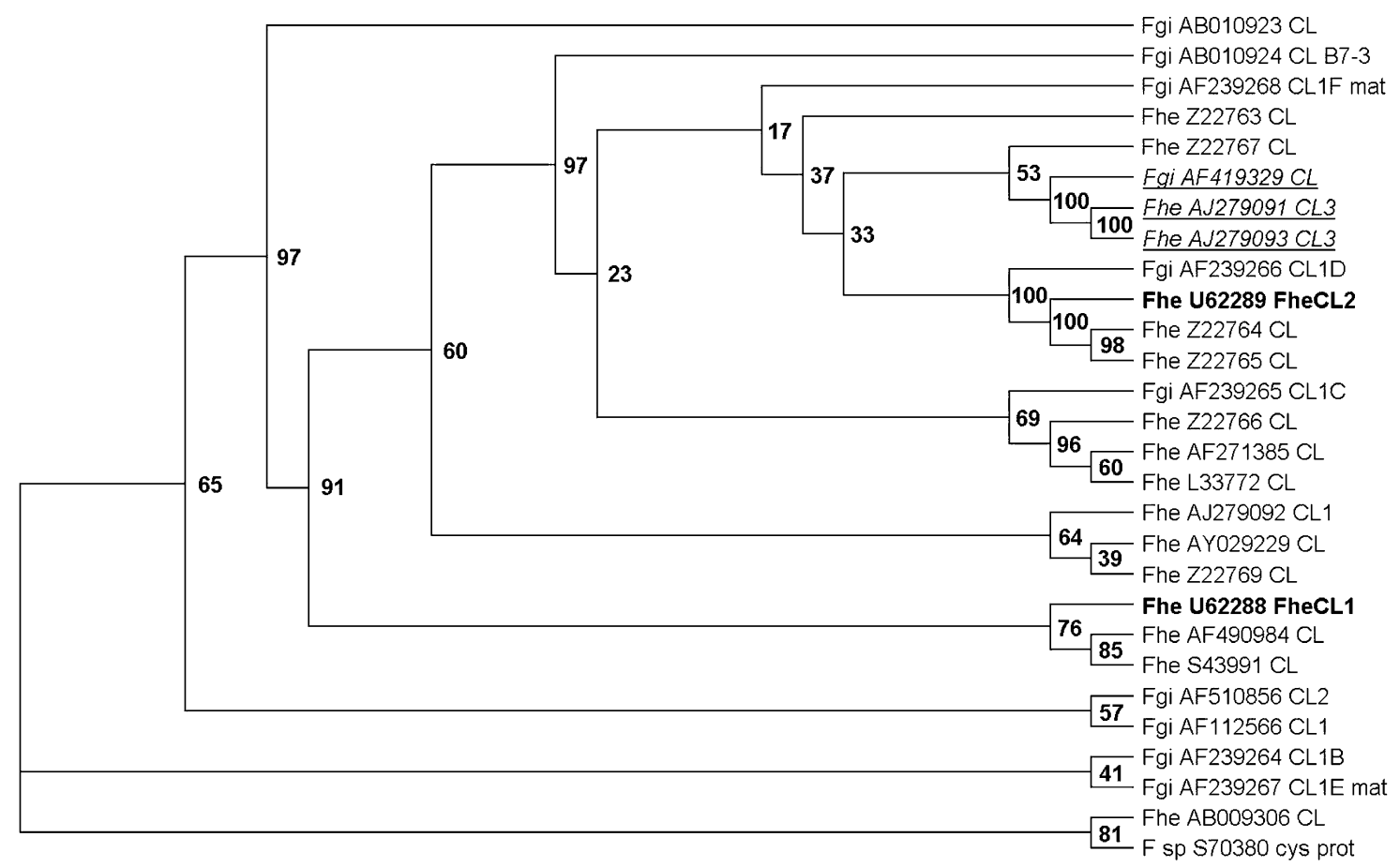

Fig. 2. Dendrogram of $F$. hepatica and F. gigantica cathepsin L gene family. Relationship of the cathepsin L cDNAs (public database accession numbers at end of branches). FheCL1 and FheCL2 are highlighted in bold. Sequences isolated from cDNA from newly excysted juveniles are italicised. Samples were analysed using 1,000 bootstrap replicates (numbers represent values obtained). 
differ in only a few amino acids and may represent alleles rather than distinct genes. Nevertheless, it is clear that at least seven different cathepsin L genes are expressed by these trematodes. Phylogenetic studies of this gene family revealed that they belong to a clade that includes the mammalian cathepsin Ls, cathepsin Ss and cathepsin Ks (the parasite enzymes show 40-55\% similarity with the mammalian homologues) (Tort et al., 1999) Of particular note is that the newly excysted juvenile (NEJ)-specific cathepsin L cDNAs (two from $F$. hepatica and one from $F$. gigantica) cluster together in the dendrogram shown in Fig. 2, which may suggest that cathepsin L expression in NEJ is limited to two genes whereas in the adult stages many more genes are expressed.

It is difficult to assess the temporal expression and localisation of each of the cathepsin $\mathrm{L}$ gene products because their close nucleic acid/amino acid sequences similarities preclude the development of specific PCR/antibody reagents. However, in situ hybridisation studies by Grams et al. (2001) with $F$. gigantica and by us with F. hepatica (Fig. 3) are consistent with immunolocalisation studies (Smith et al., 1993) and show that the vast majority of cathepsin L proteases are expressed in the cells lining the parasite's guts. It is possible that more than FheCL1 and FheCL2 proteases are secreted by the parasite, which would agree with the 2-D electrophoresis analysis by Wijffels et al. (1994) and Jefferies et al. (2001) that shows the presence of multiple cathepsin L proteases in adult $F$. hepatica ES products. The structure of one of the $F$. gigantica genes was recently determined by Yamasaki et al. (2002), and consists of four exons and three introns of similar size and arrangement to those found in crustacean and mammalian cathepsin Ls.

\section{Regulation of cathepsin $L$ activity}

Enzymatic assays have shown that cathepsin L activity is expressed in the infective stage of the parasite, the NEJ, in the immature developmental stages that migrate through the liver parenchyma and in adults that reside in the bile ducts (Carmona et al., 1993; Smith et al., 1993). A proteomics approach to characterising NEJ proteins confirmed the presence of cathepsin L proteases in this stage (Tkalcevic et al., 1995) and now full-length $F$. hepatica and $F$. gigantica NEJ-specific cathepsin L sequences are available in the public databases (Fig. 2).

As mentioned above the proteases are synthesised in the epithelial cells that line the parasite gut (Fig. 3). Within these cells the proteases are packaged into secreted vesicles in high concentration ready for secretion. During trafficking to these secretory vesicles the activity (and stability) of the cathespin Ls is controlled by the presence of the pro-peptide on the protein (as has been demonstrated for mammalian cathepsin Ls) (Roche et al., 1997). It remains unclear whether the proteases are activated before they reach the vesicles or following their secretion by the NEJ and by the adult.

It is possible that other mechanisms exist to protect the parasite's cells from unwanted protease activity. Recently, Khaznadji, Collins, Dalton and Moire (unpublished) discovered a novel molecule that contains five cystatin

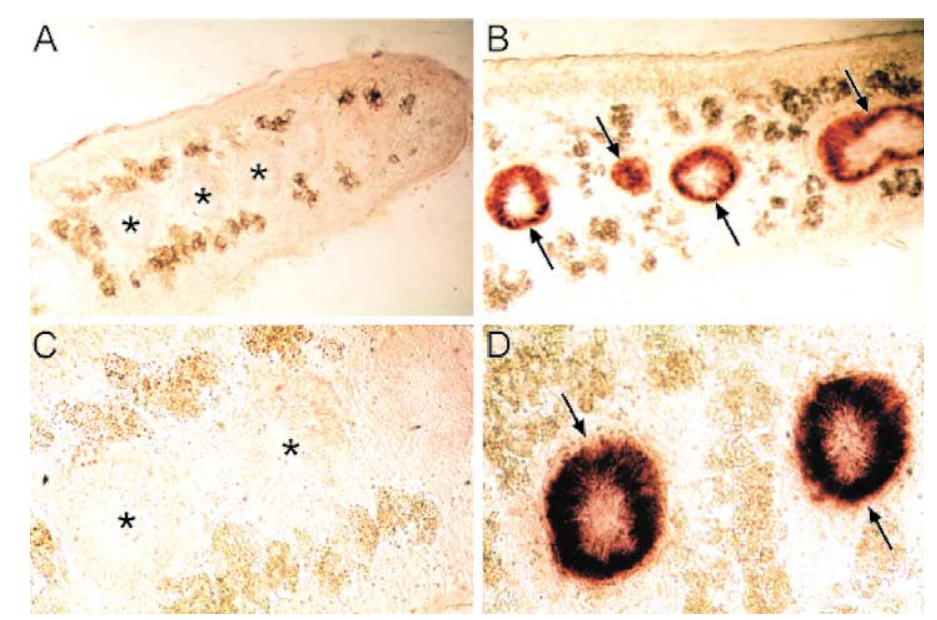

Fig. 3. Localisation of cathepsin L proteases by in situ hydridisation. Light micrographs showing the expression of cathepsin L protease in transverse sections $(20 \mu \mathrm{M})$ of adult $F$. hepatica. In situ hybridisation was performed using digoxigenin-labelled DNA probes to cathepsin L protease gene. Specific sense $\left[5^{\prime}\right.$-tgtggctcctgttgggcattc- $\left.3^{\prime}\right]$ and anti-sense $\left[5^{\prime}\right.$-ggattcggttccaatcc- $\left.3^{\prime}\right]$ primers were designed to amplify a region of $195 \mathrm{bp}$ (including primers) from $F$. hepatica cathepsin L genes. Each probe (sense strand and anti-sense strand) was generated using asymmetrical PCR and digoxigenin-labelled di-nucleotide triphosphates (dNTPs) (Roche Diagnostics); cryostat sections were stained following overnight hybridisation at $50{ }^{\circ} \mathrm{C}$. Gene expression can be seen as a dark red/brown chromogen formed by substrate cleavage by alkaline phosphatase conjugated to anti-digoxigenin antibodies bound to the probes. All sections were treated in the same way except that A and C were exposed to the sense strand probes (negative controls) whereas B and D were exposed to the anti-sense strand probes. Asterisks indicate gut lumen and arrows indicate positive staining/cathepsin L expression. (A) Low power micrograph showing unstained gut. Mag. $\times 20$. (B) Low power micrograph showing positive staining in four gut. Mag. $\times 20$. (C) High power micrograph showing several unstained gut. Mag. $\times 40$. (D) High power micrograph showing strong positive staining in 2 gut. Mag. $\times 40$. 
The cystatin families

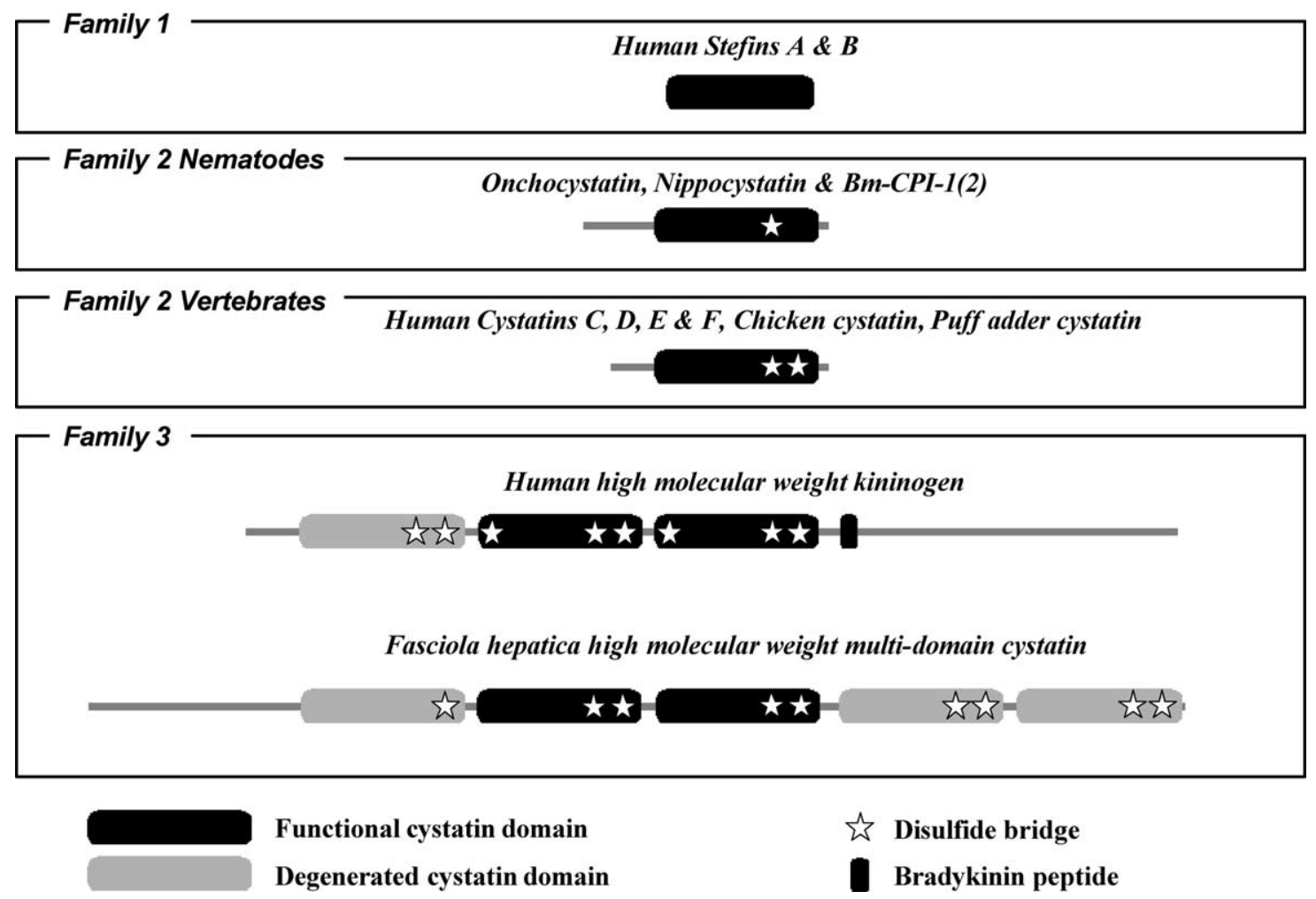

Fig. 4. The novel multi-domain F. hepatica cystatin. The Nematode cystatins-like Onchocystatin from Onchocerca volvulus, Nippocystatin from Nippostrongylus brasiliensis and Bm-CPI-1 from Brugia malayi are related to the cystatin family 2 of the cystatin superfamily (see Maizels et al., 2001). The $F$. hepatica multi-domain cystatin, however, is structurally related to the kininogen family (or cystatin family 3 ) but may be the first-described member of a new cystatin family.

domains, two of which are potent inhibitors of cathepsin $\mathrm{L}$ (the structure of the other three cystatin domains appears to be degenerate, Fig. 4). This multi-domain cystatin is expressed in NEJ and adult flukes but its expression is greater in the former stage. The cathepsin L protease may be required in large amounts by the NEJ to allow them quickly to invade the host intestinal wall following excystment, and a corresponding cystatin inhibitor may be necessary to protect the parasite from its own proteolytic activity during its storage or after secretion.

\section{Functions of the cathepsin Ls}

Cathepsin Ls that are secreted into the gut lumen following ingestion of host blood and liver tissue perform the task of digesting host tissues. The breakdown products of this endoproteolysis are then absorbed into the epithelial cells where they may undergo further degradation by dipeptidyl peptidases and aminopeptidases to amino acids that are then used in the anabolism of parasite proteins, or in the case of adults, in the production of eggs (Tort et al., 1999).

Because liver flukes possess a blind-ending intestine, the contents of the gut must be emptied by frequent regurgitation (approximately every $3 \mathrm{~h}$ ). Accordingly, the protease must reach the outside tissue where they may carry out additional important functions for the parasite, including the excavation of a migratory tract. In this context, we have demonstrated that liver fluke cathepsin Ls can efficiently cleave interstitial matrix proteins such as fibronectin, laminin and native collagen (Berasain et al., 1997). We have also shown that the parasite cathepsin Ls can cleave immunoglobulins precisely in the hinge region (thus separating the Fab from the Fc regions) and prevent the antibody-mediated attachment of eosinophils to the parasite surface and hence may aid in protecting the parasite from immune attack (Carmona et al., 1993; Smith et al., 1993; Berasain et al., 1997).

More recently, however, we have proposed that the secreted cathepsin Ls may be involved in suppression and/or modulation of Th1 immune responses and induction of nonprotective host Th2 responses. An analysis of cytokine production by antigen-stimulated spleen cells of $F$. hepaticainfected mice showed that these are predominantly of the Th2 type, i.e. production of interleukin (IL)-4, IL-5 and IL-10, but little or no IFN- $\gamma$ (O'Neill et al., 2000). This is consistent with immunological observations in cattle which show that in the early stages of infections mixed Th1/Th2 responses are observed but as infection progresses, a Th2 
Table 1

Summary of vaccine trials with $F$. hepatica cathepsin L proteases

\begin{tabular}{|c|c|c|c|c|}
\hline Antigen & Host & Dose & Protection $(\%)$ & $\begin{array}{l}\text { Reduction in } \\
\text { viable eggs }(\%)\end{array}$ \\
\hline FheCL1 ${ }^{\mathrm{a}}$ & Cattle & $10-200 \mu \mathrm{g} \times 3$ & $38.2-69.4$ & ND \\
\hline FheCL1 ${ }^{\mathrm{a}}$ & Cattle & $200 \mu \mathrm{g} \times 3$ & 43 & $40-65$ \\
\hline FheCL1 1 haem fraction $^{\mathrm{a}}$ & Cattle & $200 \mu \mathrm{g} \times 3$ & 52 & 80 \\
\hline FheCL1 + haem fraction ${ }^{\mathrm{a}}$ & Cattle & $200 \mu \mathrm{g} \times 3$ & 72.4 & 98 \\
\hline FheCL1 + FheCL1 ${ }^{\mathrm{b}}$ & Cattle & $200 \mu \mathrm{g} \times 3$ & 54 & 55 \\
\hline FheCL2 ${ }^{c}$ & Sheep & $100 \mu \mathrm{g} \times 2$ & 33 & 81 \\
\hline FheCL2 + FheCL2 ${ }^{\text {c }}$ & Sheep & $100 \mu \mathrm{g}$ each $\times 2$ & 60 & ND \\
\hline FheCL2 + FheCL2 + LAP $^{\mathrm{c}}$ & Sheep & $100 \mu \mathrm{g}$ each $\times 2$ & 79 & ND \\
\hline FheCL proteases ${ }^{\mathrm{d}}$ & Sheep & $120+90 \mu \mathrm{g}$ & 0 & 69.4 \\
\hline
\end{tabular}

ND, not determined.

a Dalton et al. (1996).

b Mulcahy et al. (1998).

c Piacenza et al. (1999).

d Wijffels et al. (1994).

response predominates (Mulcahy et al., 1999). However, at high levels of infection in mice (approximately 15 metacercariae) a marked suppression of Th1 responses was observed (O'Neill et al., 2000). Moreover, when mice were concurrently infected with $F$. hepatica and Bordetella pertussis, the Th1-specific response to the bacterial pathogen (assessed by measuring IFN- $\gamma$ ) was suppressed. This suppression led to a delay in the clearance of the bacterium in the co-infected mice as compared to mice infected with B. pertussis alone (Brady et al., 1999). Liver fluke infection also suppressed the Th1 responses to a $B$. pertussis whole cell vaccine (WCV) suggesting that the parasite may secrete Th1 suppressive factors. Subsequently, O'Neill et al. (2001) demonstrated that injection of purified cathepsin L just prior to vaccination with $B$. pertussis WCV blocked the production of IFN- $\gamma$ by WCV-stimulated spleen cells. It is possible, therefore, that secreted cathepsin Ls play some immunomodulatory/immunosuppressive function by blocking the development of protective Th1 responses in the host and thus aiding the progression of non-protective Th2 responses that would favour the longevity of the parasite.

\section{Vaccine trials with cathepsin $L$ proteases}

The involvement in biological roles that are essential to the parasite's survival in the host makes the $F$. hepatica cathepsin $\mathrm{L}$ proteases likely targets at which novel vaccines could be directed. Native FheCL1 and FheCL2 proteases could be purified from the ES products of adult worms by a series of conventional gel permeation and ion exchange chromatography steps and produced in sufficient quantities to allow experimental vaccine trials in cattle. In our first vaccine trial a dose-ranging experiment demonstrated that significant protection against liver fluke infection (average $54 \%$ ) could be obtained and that doses of as little as $10 \mu \mathrm{g}$ of protein, given on three occasions, could induce protective responses (Dalton et al., 1996). Subsequent trials, performed with $200 \mu \mathrm{g}$ per dose, confirmed the protective nature of the cathepsin L proteases (Table 1). When the cathepsin L proteases were administered in conjunction with other liver fluke antigens, such as a haem-binding protein, protection levels of $73 \%$ were achieved (Dalton et al., 1996, Table 1). A further vaccine trial in cattle showed that a combination of FheCL1 and FheCL2 elicited 53\% protection against challenge, and trials in sheep induced protection levels from 33 to $60 \%$ (Mulcahy et al., 1998; Piacenza et al., 1999, Table 1). When FheCL1 and FheCL2 were combined with $F$. hepatica leucine aminopeptidase (LAP) protection levels were $79 \%$ in sheep (Piacenza et al., 1999).

Additional beneficial aspects of the protection induced by the cathepsin $\mathrm{L}$ vaccines were observed. First, in all vaccine trials the proportion of liver flukes that did not develop to maturity was greater in vaccinates than in nonvaccinated controls. Consequently, the damage to the host's liver during acute infection was significantly reduced. Secondly, vaccination of both sheep and cattle also elicited a highly significant reduction $(50-98 \%)$ of the parasite's ability to produce eggs, and those eggs that were synthesised showed reduced 'hatch rates' (Dalton et al., 1996; Mulcahy et al., 1998; Table 1). The implications of these findings are that by reducing the parasite burden of the host and, at the same time, blocking the synthesis of viable eggs by those parasites that do survive in vaccinated animals, the vaccine would have a profound effect on pasture contamination and hence disease transmission.

\section{What is the mechanism behind protection?}

Studies examining the immune responses of cattle that were given either experimental or natural infections of $F$. hepatica metacercariae showed that animals generate IgG1 antibodies and little or no IgG2 antibodies 
(Mulcahy et al., 1999; Hoyle et al., 2002). These observations suggest that the immune responses to liver fluke antigens are highly polarised to a Th2 type. On the other hand, antibody responses to the cathepsin L-based vaccines include high titres of both $\mathrm{IgG} 1$ and $\mathrm{IgG} 2$ indicating that protection is associated with the induction of a Th1 responses or a mixed Th1/Th2 response (Mulcahy et al., 1998, 1999).

A recent analysis of the cathepsin L-specific IgG1 antibody responses in infected cattle revealed that these antibodies do not appear in the serum until 4 or 5 weeks post-infection. Moreover, animals given a second infection 4 weeks after the primary infection did not exhibit any boosting of immune responses to the cathepsin $\mathrm{L}$ proteases (Hoyle et al., 2002; Fig. 5). It is clear, therefore, that cathepsin Ls are not highly immunogenic in the early stages of infection such that, at this stage, they may be considered 'hidden antigens'. This may be an important strategy used by the parasite to prevent antibodies being generated to critical molecules. Accordingly, vaccinating animals with cathepsin Ls would elicit a high titre of anti-cathepsin L antibodies that would upset this evasion strategy and, therefore, would be detrimental to the early migratory stages of the parasite. Indeed, when animals are vaccinated with cathepsin $\mathrm{L}$ a positive correlation was observed between protection and antibody titres (Mulcahy et al., 1998; Fig. 6).

The effects of the vaccine on egg production and 'hatch rate' may be mediated by antibodies that inhibit parasite feeding by blocking cathepsin L activity, thereby preventing the acquisition of amino acids for the synthesis of egg proteins. Additionally and/or alternatively, since cathepsin Ls have been immunolocalised to the reproductive organs of the mature parasite (Spithill et al., 1999a; Wijffels et al., 1994) and may play a role in egg production,

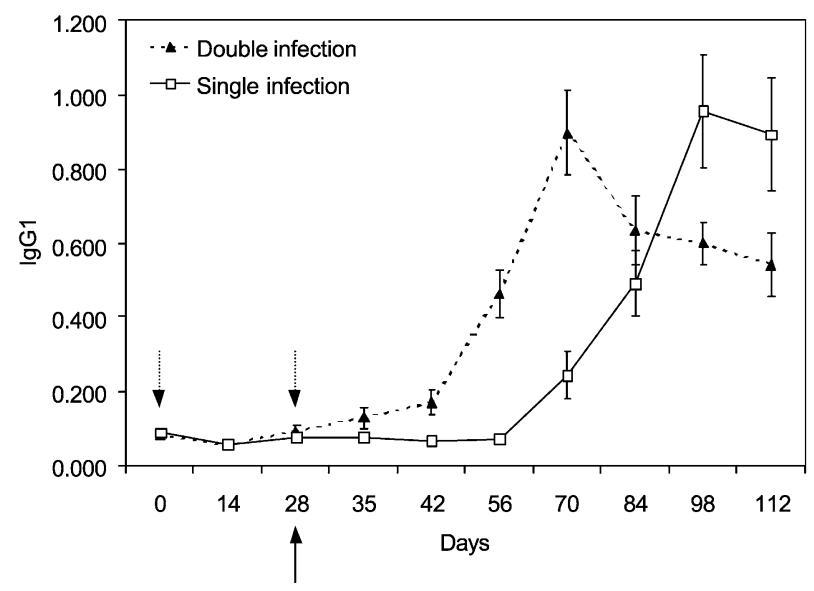

Fig. 5. IgG1 antibody response of experimental-infected cattle to FheCL1. Cattle were experimentally infected with metacercariae of $F$. hepatica either once (solid arrow) or twice (4 weeks apart, dotted arrows). Serum was collected weekly and assayed by ELISA for IgG1 antibodies to purified FheCL1 (IgG2 antibodies were either very low or undetectable). Antibody responses to FheCL1 in both experimental groups were observed 4-5 weeks after the primary infection.

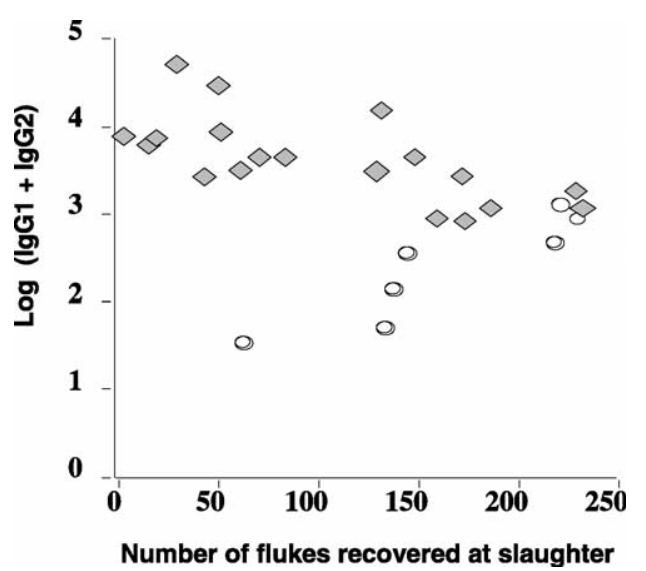

Fig. 6. Immune responses of FheCL1-vaccinated and non-vaccinated control cattle. Relationship between antibody titres and numbers of liver fluke recovered from livers of non-vaccinated animals and cattle vaccinated with cathepsin $\mathrm{L}$ proteases. In vaccinated animals, high total antibody titres (diamonds) correlate with lower fluke burdens (these antibodies consist of both IgG1 and IgG2). In contrast, in non-vaccinated control animals (circles) high antibody titres correlate with higher infections (these antibodies consist predominantly of $\operatorname{IgG} 1$, see text).

vaccine-induced antibodies may inhibit the enzyme and thereby have a direct effect on female reproductive structures.

While antibodies seem to be central to the protective effects of the cathepsin L vaccine, it is clear that the generation of a Th1 or Th1/Th2 type immune response is also critical. The induction of this type of response was dependant on the adjuvant employed in our studies i.e. Freund's Complete/Incomplete Adjuvant. However, it also seems likely that by blocking cathepsin L activity the vaccine prevents the parasite from suppressing the Th1 response of the host and from inducing the development of $\mathrm{Th} 2$ responses that are typical of natural infections.

\section{Functional expression of cathepsin $L$ proteases}

While FheCL1 and FheCL2 could be purified from liver fluke ES products in quantities that allowed the detailed analysis of their biochemical and physico-chemical properties, and their assessment as vaccine candidates, it is obvious that for their future commercialisation a method of recombinant production is necessary. The production of cathepsin L proteases in a fully functional form requires expression of the pro-mature form of the enzyme since the pro-peptide region is required for correct folding of the molecule. Prokaryotic systems of expression, e.g. E. coli, are not suitable for the production of functionally active cathepsin proteases, although these systems have been employed to express non-active proteins, such as F. gigantica cathepsin L (Yamasaki et al., 2002), which were subsequently re-folded to active form. Unfortunately, re-folding methodologies are complex, cumbersome and expensive, and serve to obviate their application in commercial production schemes. 
Yeast systems have proved more successful for the functional expression of many parasite proteases including the $F$. hepatica cathepsin Ls (Dalton et al., in press). We have reported that the yeast Saccharomyces cerevisiae, transformed with a full-length FheCL1 or FheCL2 cDNA, expressed and secreted functional enzyme into the culture medium from which homogeneous enzyme could be obtained by conventional purification techniques such as gel filtration and ion exchange chromatography (Dowd et al., 1997; Roche et al., 1997). While the functional expression of mammalian cathepsin proteases in S. cerevisiae has also been reported, these have necessitated the use of an $\alpha$-factor pre- or pre-pro-signals. However, functional expression of FheCL1 and FheCL2 did not require such a fusion protein and $\mathrm{N}$-terminal sequencing of expressed proteases revealed that they were processed in yeast to the mature enzyme by cleavage at the precise position as that observed for the native enzymes (Dowd et al., 1997; Roche et al., 1997). Notwithstanding these advances, the expression of cathespin L protease was low (approximately $100 \mu \mathrm{g} / \mathrm{l}$ ) and hence still not suitable for large-scale production. More recently, however, we have employed the yeast Pichia pastoris for protease expression using vectors that include the yeast $\alpha$-factor pre-pro-signals (Invitrogen). High-level expression of FheCL1 has been achieved $(800 \mathrm{mg} / \mathrm{l})$ and the protease, which carries a $\mathrm{His}_{6}$ tag, can be isolated by a onestep affinity chromatography procedure on Ni-NTA-agarose (Fig. 1). The protease is expressed as a pro-enzyme together with a semi-processed form, both of which can be activated at low $\mathrm{pH}$ to fully active mature enzyme (Fig. 1). The physico-chemical properties (substrate specificity, $\mathrm{pH}$ optima, stability, etc.) and molecular size of cathespin L are similar to the native enzyme, and more importantly with respect to vaccines, no post-translational glycosylation occurs (unpublished data). These experiments demonstrate that liver fluke FheCL1 and FheCL2 could be produced at a commercial level using yeast as the heterologous expression system.

\section{Concluding remarks}

In our laboratory the cathepsin $\mathrm{L}$ proteases of $F$. hepatica have given consistent positive results when used as vaccines against fasciolosis in cattle and sheep and therefore have excellent prospects for the development of first generation anti-fluke vaccines. Vaccinations in sheep with a purified fraction of cysteine proteases of adult $F$. hepatica carried out by Wijffels et al. (1994), however, did not provide any protection against a challenge infection, although a $>70 \%$ reduction of faecal eggs counts (FEC) was observed (Table 1). In addition, vaccination trials in cattle with $F$. gigantica cathepsin Ls did not protect animals against this fluke species (Spithill et al., 1999a). The discrepancies remain unresolved and may only be resolved if a comparative analysis of the immune responses to the vaccines was carried out. There are differences in vaccine formulations, doses and/or route of administration between the trials (see Spithill et al., 1999a,b) that may have induced a variety of effector mechanisms to the vaccines. Each of these factors will need to be investigated systematically during the developmental stages of a commercial vaccine.

Besides the cathepsin $\mathrm{L}$ proteases there are several other molecules that are potential vaccine candidates including GST, FABP (Spithill and Dalton, 1998) and LAP (Piacenza et al., 1999). While trials with these antigens have given some very variable results, no detailed analysis of immune responses to the vaccines has been carried out to determine immunological factors that correlate with protection/no protection. It is therefore still feasible that these molecules could be realised as a basis for commercial vaccines, either alone or in combination with other candidates, if the precise conditions for inducing protection were elucidated.

The commercialisation of a vaccine will require a system for production and downstream processing that is not so costly as to reduce the final product's competitiveness against current drug treatments. Both E. coli and yeast expression systems may be suitable for the production of vaccines for experimental trials, but before progressing into developmental phase the best/cheapest system for up-scale production/downstream processing will have to be considered for final commercialisation in an animal health market that already suffers from relatively low-valued/low profit-margin products (Dalton et al., in press). Nevertheless, the prospects for future vaccines are good, particularly considering the pressure on the industry to move away from chemical treatments.

\section{References}

Andrews, S.J., 1999. The life cycle of Fasciola hepatica. In: Dalton, J.P., (Ed.), Fasciolosis, CAB International, Wallingford, UK, pp. 1-30.

Anon., 18 March 1995. Resistance to fluke drug found on Sligo farm. Irish Farmer's J. 2

Barrett, A.J., Kirschke, H., 1981. Cathespin B, cathepsin H and cathepsin L. Methods Enzymol. 80, 535-561.

Berasain, P., Goni, F., McGonigle, S., Dowd, A., Dalton, J.P., Frangione, B., Carmona, C., 1997. Proteinases secreted by Fasciola hepatica degrade extracellular matrix and basement membrane components. J. Parasitol. 83, 1-5.

Brady, M.T., O’Neill, S.M., Dalton, J.P., Mills, K.H.G., 1999. Fasciola hepatica suppresses protective Th1 responses against Bordetella pertussis. Infect. Immun. 67, 5372-5378.

Carmona, C., Dowd, A.J., Smith, A.M., Dalton, J.P., 1993. Cathespin L proteinase secreted by Fasciola hepatica in vitro prevents antibodymediated eosinophil attachment to newly excysted juveniles. Mol. Biochem. Parasitol. 62, 9-18.

Dalton, J.P., Heffernan, M., 1989. Thiol proteases released in vitro by Fasciola hepatica. Mol. Biochem. Parasitol. 35, 161-166.

Dalton, J.P., McGonigle, S., Rolph, T.P., Andrews, S.J., 1996. Induction of protective immunity in cattle against infection with Fasciola hepatica by vaccination with cathepsin $\mathrm{L}$ proteinase and haemoglobin. Infect. Immun. 64, 5066-5074. 
Dalton, J.P., Brindley, P.J., Knox, D.P., Hotez, P.J., Brady, C.P., Donnelly, S., O'Neill, S.M., Mulcahy, G., Loukas, A., 2003. Helminth vaccines: from mining genomic information for vaccine targets to systems used for protein expression. Int. J. Parasitol, 33, 621-640.

Dowd, A.J., Dooley, M., Fagain, C., Dalton, J.P., 2000. Stability studies on the cathepsin L proteinase of the helminth parasite, Fasciola hepatica. Enzyme Microb. Technol. 27, 599-604.

Dowd, A.J., Smith, A.M., McGongle, S., Dalton, J.P., 1994. Purification and characterisation of a second cathepsin $\mathrm{L}$ proteinase secreted by the trematode Fasciola hepatica. Eur. J. Biochem. 223, 91-98.

Dowd, A.J., Tort, J., Roche, L., Dalton, J.P., 1997. Isolation of a cDNA encoding cathepsin L2 and functional expression in Saccharomyces cerevisiae. Mol. Biochem. Parasitol. 88, 163-174.

Fairweather, I., Boray, J.C., 1999. Mechanism of fasciolicide action and drug resistance in Fasciola hepatica. In: Dalton, J.P., (Ed.), Fasciolosis, CAB International, Wallingford, UK, pp. 225-276.

Gaasenbeek, C.P.H., Moll, L., Cornelissen, J.B.W.J., Vellema, P., Borgsteede, F.H.M., 2001. An experimental study on triclabendazole resistance of Fasciola hepatica in sheep. Vet. Parasitol. 95, 37-43.

Grams, R., Vichasri-Grams, S., Sobhorn, P., Upatham, E.S., Viyanant, V., 2001. Molecular cloning and characterisation of cathepsin L encoding genes from Fasciola gigantica. Parasitol. Int. 50, 105-114.

Heussler, T.V., Dobbelaere, A.E.D., 1994. Cloning of a protease gene family of Fasciola hepatica by the polymerase chain reaction. Mol. Biochem. Parasitol. 64, 11-23.

Hoyle, D.V., Dalton, J.P., Chase-Topping, M., Taylor, D.W., 2002. Preexposure of cattle to drug-abbreviated Fasciola hepatica infections: the effect upon subsequent challenge infection and early immune response. Vet. Parasitol. 111, 65-82.

Jefferies, J.R., Campbell, A.M., van Rossum, A.J., Barrett, J., Brophy, P.M., 2001. Proteomic analysis of Fasciola hepatica excretory-secretory products. Proteomics 1, 1128-1132.

Mas-Coma, S., Bargues, M.D., Esteban, J.G., 1999. Human fasciolosis. In: Dalton, J.P., (Ed.), Fasciolosis, CAB International, Wallingford, UK, pp. 411-434.

Maizels, R.M., Gomez-Escobar, N., Gregory, W.F., Murray, J., Zang, X., 2001. Immune evasion genes from filarial nematodes. Int. J. Parasitol. $31,889-898$

Mitchell, G.B.B., Maris, L., Bonniwell, M.A., 1998. Triclabendazoleresistant liver fluke in Scottish sheep. Vet. Rec. 143, 399.

Moll, L., Gassenbeek, C.P.H., Vellema, P., Borgsteede, F.H.M., 2000. Resistance of Fasciola hepatica against triclabendazole in cattle and sheep in the Netherlands. Vet. Parasitol. 91, 153-158.

Mulcahy, G., O'Connor, F., McGonigle, S., Dowd, A.J., Clery, D., Andrews, S.J., Dalton, J.P., 1998. Correlation of specific antibody titre and avidity with protection in cattle immunized against Fasciola hepatica. Vaccine 16, 932-939.

Mulcahy, G., Joyce, P., Dalton, J.P., 1999. Immunology of Fasciola hepatica infection. In: Dalton, J.P., (Ed.), Fasciolosis, CAB International, Wallingford, UK, pp. 341-376.
O’Neill, S.M., Parkinson, M., Strauss, W., Angles, R., Dalton, J.P., 1998. Immunodiagnosis of Fasciola hepatica infection (fascioliasis) in a human population in the Bolivian altiplano using purified cathepsin $\mathrm{L}$ cysteine protease. Am. J. Trop. Med. Hyg. 58, 417-423.

O'Neill, S.M., Brady, M.T., Callanan, J.J., Mills, K.H.G., Dalton, J.P., 2000. Fasciola hepatica infection down-regulates Th1 response in mice. Parasite Immunol. 22, 147-155.

O'Neill, S.M., Mills, K.H.G., Dalton, J.P., 2001. Fasciola hepatica cathepsin L cysteine proteinase suppresses Bordetella pertussis-specific interferon- $\gamma$ production in vivo. Parasite Immunol. 23, 541-547.

Overend, D.J., Bowen, F.L., 1995. Resistance of Fasciola hepatica to triclabendazole. Aust. Vet. J. 72, 275-276.

Piacenza, L., Acosta, D., Basmadjian, I., Dalton, J.P., Carmoma, C., 1999. Vaccination with cathepsin $\mathrm{L}$ proteinases and with leucine aminopeptidase induces high levels of protection against fascioliasis in sheep. Infect. Immun. 67, 1954-1961.

Roche, L., Dowd, A.J., Tort, J., McGonigle, S., McSweeney, A., Curley, P., Ryan, T., Dalton, J.P., 1997. Functional expression of Fasciola hepatica cathepsin L1 in Saccharomyces cerevisiae. Eur. J. Biochem. 245, 373-380.

Rokni, M.B., Massoud, J., O’Neill, S.M., Parkinson, M., Dalton, J.P., 2002. Diagnosis of human fasciolosis in the Gilan province of northern Iran: application of cathepsin L-ELISA. Diagn. Microbiol. Infect. Dis. 44, $175-179$.

Smith, A.M., Dowd, A.J., McGonigle, S., Keegan, P.S., Brennan, G., Trudgett, A., Dalton, J.P., 1993. Purification of a cathepsin L proteinase secreted by adult Fasciola hepatica. Mol. Biochem. Parasitol. 62, 1-8.

Spithill, T.W., Dalton, J.P., 1998. Progress in the development of liver fluke vaccines. Parasitol. Today 14, 224-228.

Spithill, T.W., Smooker, P.M., Sexton, J.L., Bozas, E., Morrison, C.A., Creaney, J., Parsons, J.C., 1999a. Development of vaccines against Fasciola hepatica. In: Dalton, J.P., (Ed.), Fasciolosis, CAB International, Wallingford, UK, pp. 377-401.

Spithill, T.W., Smooker, P.M., Copeman, D.B., 1999b. Fasciola gigantica: epidemiology, control, immunology and molecular biology. In: Dalton, J.P., (Ed.), Fasciolosis, CAB International, Wallingford, UK, pp. 465-526.

Tkalcevic, J., Ashman, K., Meeusen, E.N.T., 1995. Fasciola hepatica: rapid identification of newly excysted juvenile proteins. Biochem. Biophys. Res. Commun. 213, 169-174.

Tort, J., Brindley, P.J., Knox, D., Wolfe, K.H., Dalton, J.P., 1999. Helminth proteinases and their associated genes. Adv. Parasitol. 43, 161-266.

Wijffels, G.L., Salvatore, L., Dosen, M., Waddington, J., Thompson, C., Campbell, N., Sexton, J., Wicker, J., Bowen, F., Friedel, T., Spithill, T.W., 1994. Vaccination of sheep with purified cysteine proteinases of Fasciola hepatica decreases worm fecundity. Exp. Parasitol. 78, $132-148$.

Yamasaki, H., Mineki, R., Murayama, F., Ito, A., Aoki, T., 2002. Characterisation and expression of the Fasciola gigantica cathepsin $\mathrm{L}$ gene. Int. J. Parasitol. 32, 1031-1042. 